\title{
Variación genecológica de dos poblaciones contiguas de Drimys winteri (Forst.)
}

\author{
Genecological variation of two contiguous populations of Drimys winteri (Forst.)
}

MARCELO HERNANDEZ ${ }^{1}$, CLAUDIO DONOSO², MAGDALENA ROMERO 3

${ }^{1}$ Departamento de Ciencias Forestales, Universidad Católica de Temuco, Casilla 15-D, Temuco, Chile.

${ }^{2}$ Instituto de Silvicultura. ${ }^{3}$ Instituto de Botánica, Universidad Austral de Chile, Casilla 567, Valdivia, Chile.

\begin{abstract}
SUMMARY
Two adjacent populations of Drimys winteri (Forst.) were found on the Andean western slopes of Parral. The fact that they were clearly different and had different habitat gave rise to the question concerning their taxonomy; the hypothesis of a possible genecological differentiation between both populations was stated. Research studies on the morphological and chemical characteristics as well as on the ecophysiological behaviour of both common canelo populations and variant canelo populations were carried out.

These results have allowed the verification of the existence of two ecotypes in the Drimys winteri var. chilensis species.
\end{abstract}

Key words: genecological variation, Drimys winteri, ecotypes, populations.

\section{RESUMEN}

En la precordillera andina de Parral se encontraron dos poblaciones contiguas de canelo (Drimys winteri Forst.), las que eran claramente diferentes a simple vista y que ocupaban hábitats distintos. Debido a esto surgió la interrogante acerca de su taxonomía y se planteó la hipótesis de una posible diferenciación genecológica entre ambas poblaciones.

Para dilucidar esta probable variación se efectuaron estudios de las características morfológicas y químicas, y del comportamiento ecofisiológico de las poblaciones del canelo común y de las poblaciones de individuos variantes. Los resultados obtenidos han permitido comprobar en la variedad Drimys winteri var. chilensis la existencia de dos ecotipos o razas ecológicas.

Palabras claves: variación genecológica, Drimys winteri, ecotipos, poblaciones.

\section{INTRODUCCION}

Canelo (Drimys winteri) es una especie que ha experimentado numerosos cambios en lo que se refiere a su ordenamiento taxonómico. Muñoz (1959) y Muñoz (1980) identifican una sola especie (Drimys winteri Forst.) con cuatro variedades distribuidas en el país. Posteriormente Troncoso et al. (1980) y Rodríguez et al. (1983) identifican una especie continental (Drimys winteri Forst.) y una especie endémica del Archipiélago de Juan Fernández (Drimys confertifolia Phil). Para la especie continental caracteriza además tres varieda- des: Drimys winteri Forst. var. chilensis, Drimys winteri var. punctata (LAM) D.C. y Drimys winteri var. andina Reiche. Finalmente Rodríguez y Quezada (1991), en la nueva y última clasificación, elevan a rango específico a $D$. winteri var. andina, clasificándola como especie, pasándose a denominar actualmente Drimys andina (Reiche) R.A. Rod. et. Quez.

No debe sorprender esta gran variabilidad de la especie ya que cumple con tres factores que posibilitan la variabilidad: una amplia distribución geográfica, una distribución ecológica muy discontinua y factores del medio ambiente muy variables 
(Heslop-Harrison 1964, Spurr y Barnes 1980, Donoso 1993). Efectivamente canelo se ubica entre $\operatorname{los} 31^{\circ}$ y $\operatorname{los} 56^{\circ} \mathrm{L}$ at. S., especialmente en el territorio chileno y desde áreas a nivel del mar, hasta los límites de la vegetación arbórea en la Cordillera de los A ndes (U rban 1934, Torres 1971, Donoso 1974, Rodríguez et al. 1983). A demás en su rango de distribución crece en diferentes tipos de substrato, que varían desde suelos con muy mal drenaje, como hualves, mallines y ñadis, hasta suelos con material volcánico grueso o gravas de ríos.

Junto a la evidente variabilidad morfológica y fenológica de la especie, que la ha llevado a diferenciar variedades y luego especies distintas, se ha encontrado una variación extremadamente alta en términos de crecimiento diametral, en altura y volumétrico de la especie, lo que es atribuido a diferencias sustanciales de calidad del sitio (Corvalán 1986). Estudios específicos de diferenciación genecológica se han realizado en poblaciones de canelo ubicadas en diferentes situaciones topográficas y edáficas en la provincia de $V$ aldivia, los que han definido dos ecotipos (M illanao 1984).

Es posible que gran parte de la enorme variabilidad que se presenta en las poblaciones de canelo corresponda a diferenciación genecológica, de tipo discontinuo o ecotípico dada la clara discontinuidad de la especie. Pero también suele encontrarse diferenciación ecotípica entre poblaciones contiguas de una especie. Ese es el caso que se postula para poblaciones de la precordillera andina de la zona mesomórfica de clima mediterráneo, aproximadamente entre los $36^{\circ}$ y los $37^{\circ}$ latitud S. Entre estas latitudes, en un área cercana a la localidad y embalse Bullileo, se encontraron dos poblaciones de canelo creciendo relativamente próximas una de otra. Una de ellas se ubica en sitios más o menos planos y muy húmedos, donde los individuos de canelo crecen junto a especies mirtáceas y presentan características morfológicas típicas del canelo común. La segunda población se presenta en terrenos de topografía más abrupta, en suelos con buen drenaje y asociada con especies de Nothofagus obliqua, Nothofagus glauca, y a veces Nothofagus alpina. Los individuos de canelo de esta población presentan características fenotípicas extraordinariamente diferentes de las del canelo común, lo que sugiere la ocurrencia de un probable ecotipo.

El objetivo de este trabajo es comparar dos poblaciones contiguas de Drimys que a simple vista son diferentes y que además se encuentran en hábitats distintos. Para eso se analizan algunos caracteres morfológicos de las hojas, flores, corteza. También se analizan algunas características fisiológicas y químicas de las hojas.

\section{MATERIAL Y METODOS}

El área de estudio se ubica en las cercanías del Embalse Bullileo ( $36^{\circ} 20^{\prime} \mathrm{S}$ y $\left.71^{\circ} 22^{\prime} \mathrm{O}\right)$, aproximadamente a $700 \mathrm{~m}$ s.n.m (figura 1).

El clima de Bullileo corresponde al tipo mediterráneo templado húmedo según la clasificación de Di Castri y Hajek (1976). La topografía es montañosa y los suelos corresponden a materiales volcánicos del tipo trumaos profundos. La vegetación se enmarca dentro del tipo forestal RobleHualo (Donoso 1981).

Para determinar las diferencias entre los ejemplares de la especie de ambas poblaciones, y sobre la base de ellas inferir la ocurrencia de diferenciación ecotípica, se analizaron las características del hábitat y características morfológicas, ecofisiológicas y químicas.

Caracterización del habitat. EI hábitat se caracterizará a través de la descripción de la topografía, del contenido de humedad del suelo y de la composición florística en ambas situaciones.

CARACTERISTICAS MORFOLOGICAS

a) Foliares. Las características morfológicas estudiadas fueron la corteza, la superficie foliar a través del método gravimétrico (Steubing 1965), el largo, ancho y relación largo/ancho en cincuenta hojas de cada procedencia. Simultáneamente se midieron cuocientes de dimensión como el desarrollo de la superficie foliar ( $\mathrm{dm}^{2} / \mathrm{g}$ p.f.) y carácter esclerófilo (g p.s. $/ \mathrm{dm}^{2}$ ). A mbos son buenos indicadores de la resistencia constitucional a la sequía, y por consiguiente de las disponibilidades hídricas que presenta el medio en que se desarrolla el vegetal (Stocker 1956). Puesto que se postula que los hipotéticos ecotipos representan hábitat de distintas condiciones de humedad, se espera que exista correlación entre ellas y las características de tipo morfológicas analizadas.

b) Florales. Debido a que la estructura floral no es modificada por el ambiente y entrega valiosa información de tipo taxonómico, también se analizaron como características morfológicas al gunos elementos florales como número de pedúnculos y flores por inflorescencia y el número de sépa- 


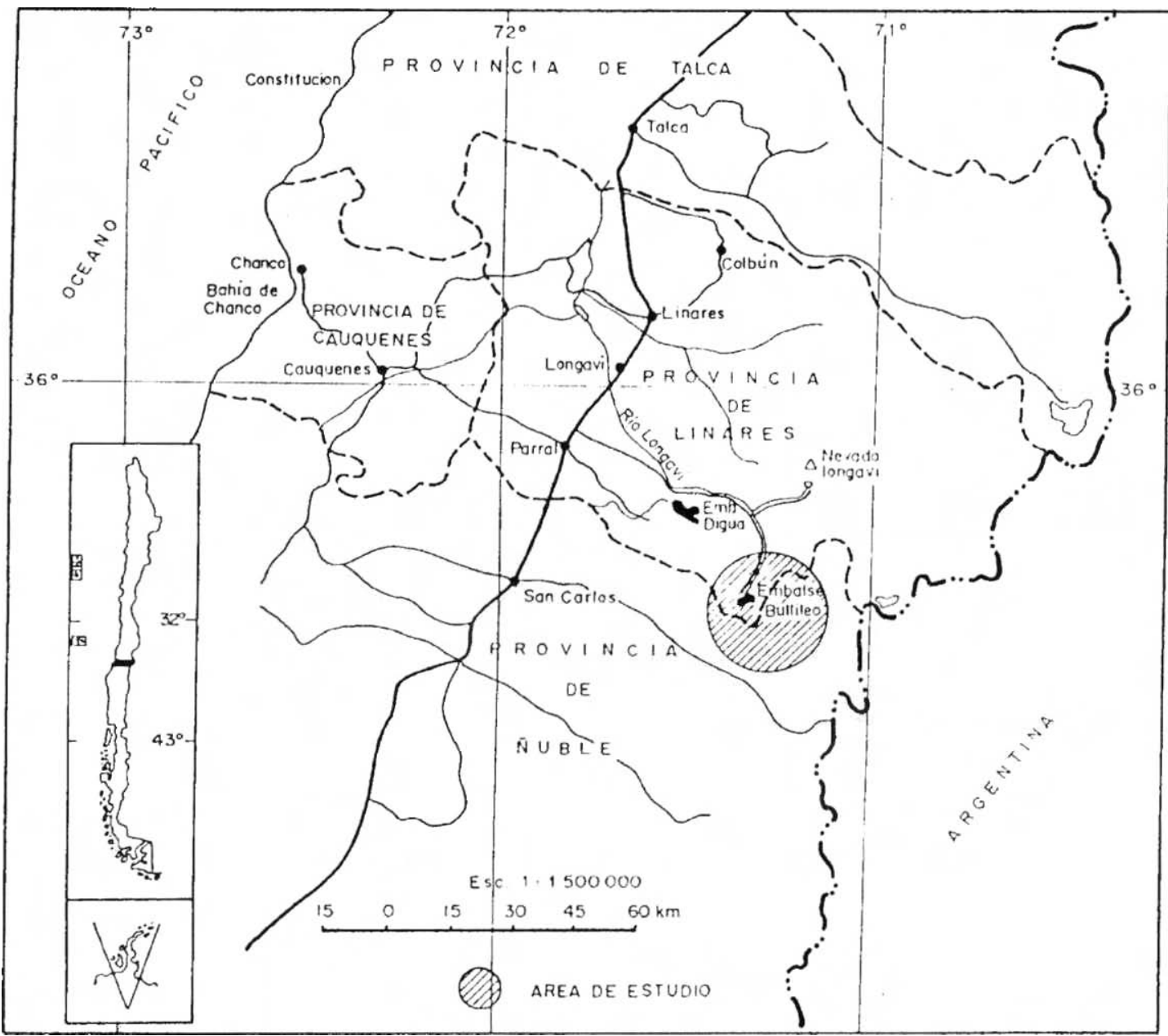

Figura 1. Ubicación geográfica del lugar de estudio.

Location of study area.

Ios, pétalos, estambres y de carpelos por flor. Para evaluar las diferencias foliares y florales se confeccionaron gráficos poligonales (Bell 1968). Se determinó también el número de semillas por kilo de la especie en estudio y se comparó con las cifras obtenidas para la especie en otros hábitats y procedencias.

Parámetros ecofisiológicos. La caracterización ecofisiológica de la vegetación consistió en el análisis de la transpiración momentánea y relativa, y la determinación del déficit crítico subletal, que según Stocker (1956) corresponde a la máxima cantidad de agua que puede perder un tejido vegetal sin sufrir daños letales. Este parámetro indica la resistencia a la sequía que posee el plasma (W einberger et al. 1972). La correlación entre las características morfológicas y fisiológicas es una forma de demostrar diferenciación genecológica (Harberd 1956, Heslop-Harrison 1964, Spurr y Barnes 1973, Donoso 1979).

ANALISIS QUIMICO

a) Ceras epicuticulares. Las hojas del canelo variante carecen del color glauco o blanquecino característico del canelo común. Por ello se analizó la cantidad de ceras epicuticulares en hojas de ambas muestras, extrayéndolas con acetona y los resultados se expresaron en peso de cera por superficie foliar.

b) Determinación de flavonoides. El análisis cromatográfico constituye una valiosa herramienta en el estudio de las relaciones taxonómicas entre individuos a través de la identificación de com- 
puestos químicos específicos, entre los cuales se encuentran los flavonoides. Estos compuestos fenólicos fueron extraídos de hojas secas y molidas, provenientes de ambas poblaciones (Heywood 1967, Donoso y $L$ andrum 1976). La extracción se realizó con solventes polares y semipolares. Los flavonoides aislados se identificaron por cromatografía bidimensional, usando luz ultravioleta y vapores de amoníaco como reveladores (A bbot y A ndrews 1970, Domínguez 1975), y espectroscopia infrarro$\mathrm{ja}$, empleando un rango de frecuencia entre 1.400 $\mathrm{cm}^{-1}$ hasta $1.800 \mathrm{~cm}^{-1}$.

Los datos de las características morfológicas y ecofisiológicas fueron sometidos a las pruebas de Kolmogorov-Smirnov (Ostle 1968) y de Bartlet (Ostle 1968, Sokal y Rohlf 1979) para verificar los supuestos de normalidad y homocedasticidad, respectivamente, con un nivel de significación de $5 \%$. Cumplidos ambos supuestos, se aplicó un análisis de varianzas, utilizándose el programa SPSS (Statiscal Packager for the Social Sciences), procedimiento Oneway ( $\mathrm{N}$ ie et al. 1975) con $1 \mathrm{y}$ $5 \%$ de nivel de significación.

A los valores del Déficit Crítico Subletal (D.C.S.) se les aplicó un Análisis de Regresión (Ostle 1968).

\section{RESULTADOS Y DISCUSION}

Características del hábitat. Las condiciones topográficas en que se desarrollan ambas poblaciones son radicalmente opuestas. La población de canelo común se ubica en terreno plano con evidente mal drenaje, como lo muestra la presencia de agua sobre la superficie del suelo en período de verano.

La población de canelo variante crece en topografía de lomaje y cerros, donde se aprecian buen drenaje y condiciones secas en la superficie del suelo, durante la estación seca. La flora de ambos hábitats posee algunas especies comunes como Nothofagus alpina, Persea lingue, Lomatia dentata, Chasquea culeuo, Pseudopanax laetevirens $y$ Lapageria rosea (cuadro 1). Sin embargo, el hábitat del canelo común se caracteriza por la abundancia de especies indicadoras de alta humedad en el suelo, como son las Mirtáceas Legrandia conccina, Liana apiculata y Myrceugenia lanceolata. La composición florística del hábitat del canelo variante, en cambio, se caracteriza por las dominancia de Nothofagus glauca y Nothofagus obliqua.

\section{CUADRO 1}

Composición florística de los hábitats de los dos tipos de canelos estudiados. Floristic composition of the habitats of two kinds of canelo.

\begin{tabular}{|c|c|c|}
\hline Arboles & $\begin{array}{l}\text { Canelo } \\
\text { común }\end{array}$ & $\begin{array}{l}\text { Canelo } \\
\text { variante }\end{array}$ \\
\hline Aextoxicon punctatum & - & * \\
\hline Cryptocaria alba & * & - \\
\hline Dasyphillum diacantoides & * & - \\
\hline Drimys winteri & * & * \\
\hline Embothrium coccineum & - & $*$ \\
\hline Gevuina avellana & - & * \\
\hline Lomatia dentata & * & * \\
\hline Luma apiculata & * & - \\
\hline Myrceugenia lanceolata & $*$ & - \\
\hline Nothofagus dombeyi & * & - \\
\hline Nothofagus alpina & * & * \\
\hline Nothofagus glauca & - & $*$ \\
\hline Nothofagus obliqua & - & * \\
\hline Persea lingue & * & * \\
\hline Podocarpus saligna & * & - \\
\hline \multicolumn{3}{|l|}{ Arbustos } \\
\hline Aristotelia chilensis & - & $*$ \\
\hline Azara petiolaris & - & * \\
\hline Chusquea culeuo & * & $*$ \\
\hline Chusquea sp. & - & $*$ \\
\hline Legrandia conccina & * & - \\
\hline Pseudopanax laetevirens & * & * \\
\hline Rhaphitamus spinosus & * & - \\
\hline \multicolumn{3}{|l|}{ Enredaderas } \\
\hline Boquilia trifoliolata & $*$ & - \\
\hline Lapageria rosea & * & * \\
\hline Hydrangea integerrina & * & - \\
\hline \multicolumn{3}{|l|}{ Arbustos } \\
\hline Blechnum hastatum & $*$ & - \\
\hline Blechnum sp. & * & $*$ \\
\hline
\end{tabular}

$*$ = presente

- = ausente

\section{CARACTERISTICAS MORFOLOGICAS}

a) Corteza. Las cortezas de ambos canelos son claramente diferentes: la del canelo variante es muy rugosa y agrietada y de color café oscuro, contrastando con la del canelo común, que es lisa, delgada y de color gris claro (fig. 2). Al romper la 


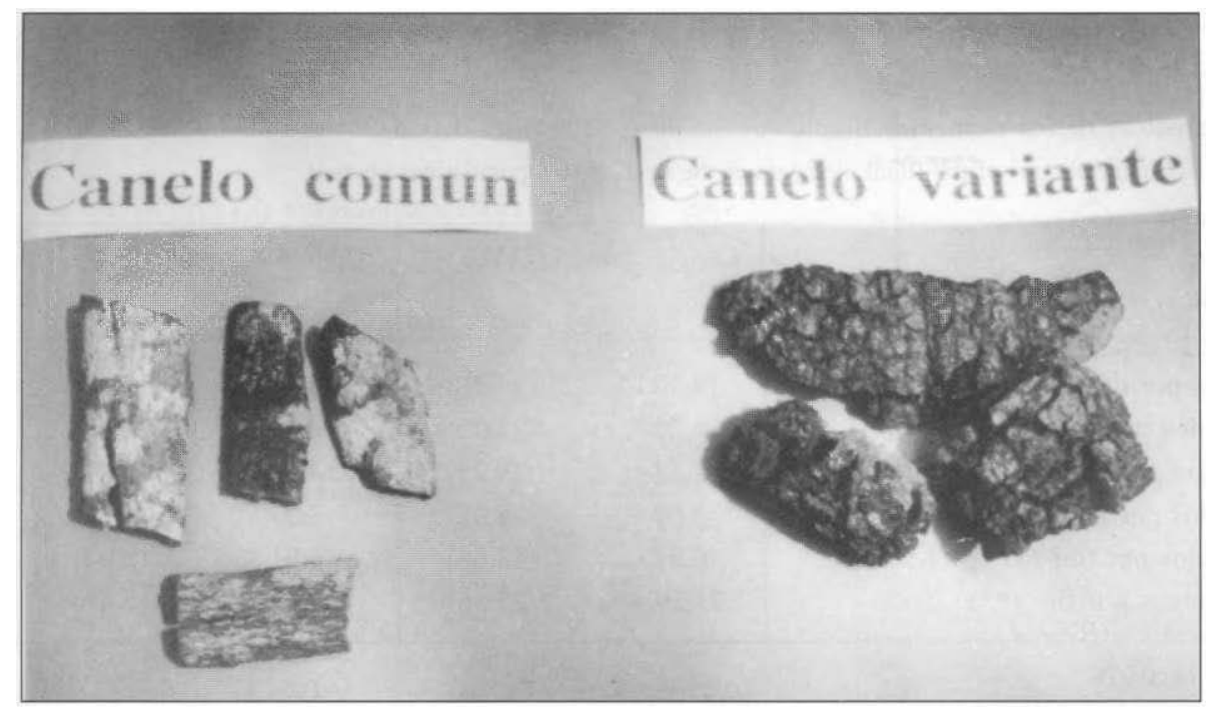

Figura 2. Cortezas de ambos canelos.

Barks from both canelos.

corteza de canelo es típico que fluya un líquido irritante y de sabor picante, lo que es una característica de la especie (Donoso 1974, Hoffmann 1982). El líquido del canelo variante, en cambio, no es picante ni irritante. No se logró determinar el compuesto químico que produce esta notable diferencia.

b) Hojas. Las dos poblaciones estudiadas difirieron en el largo y ancho, en la relación largo/ ancho, en la superficie foliar, en la densidad estomática (número de estomas $/ \mathrm{cm}^{2}$ ), en el desarrollo del área foliar y en el carácter esclerófilo (cuadro 2).

Las diferencias observadas en estas características foliares entre ambos tipos de canelo deberían indicar una adaptación del canelo variante a una condición medioambiental más seca. El mayor grado de xeromorfía del canelo variante respecto al canelo común, representado por hojas más pequeñas, de menor superficie foliar y menor desa-

\section{CUADRO 2}

Características morfológicas foliares de los dos tipos de canelos estudiados. Foliar morphological characteristics of the two kinds of canelos studied.

\begin{tabular}{|c|c|c|c|c|}
\hline \multirow{2}{*}{$\begin{array}{l}\text { Valores promedios de las } \\
\text { variables morfológicas } \\
\text { Superficie foliar }\left(\mathrm{dm}^{2}\right)\left({ }^{* *}\right)\end{array}$} & \multicolumn{2}{|c|}{ Canelo común (C.V.) } & \multicolumn{2}{|c|}{ Canelo variante (C.V.) } \\
\hline & 0.52 & $(19.0)$ & 0.43 & $(20.0)$ \\
\hline $\operatorname{Largo}(\mathrm{cm})(* *)$ & 14.01 & $(9.0)$ & 12.22 & (12.0) \\
\hline Ancho $(\mathrm{cm})(* *)$ & 4.75 & $(16.0)$ & 4.44 & (12.0) \\
\hline Relación largo/ancho $(* *)$ & 2.97 & $(10.0)$ & 2.81 & (19.0) \\
\hline Desarrollo área foliar $\left(\mathrm{dm}^{2} / \mathrm{g}\right)(*)$ & 0.26 & $(18.0)$ & 0.19 & (8.0) \\
\hline Carácter esclerófilo $\left(\mathrm{g} / \mathrm{dm}^{2}\right) \quad(*)$ & 1.52 & $(17.0)$ & 1.66 & (9.0) \\
\hline 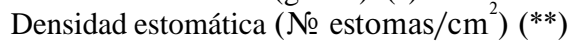 & 10.490 & $(13.0)$ & 14.320 & (9.0) \\
\hline
\end{tabular}

\footnotetext{
$(* *) \quad=$ muy significativo $(\mathrm{p}<0.001)$

$(*) \quad=$ significativo $(\mathrm{p}<0.05)$

(C.V.) $=$ coeficiente de variación (\%)
} 


\section{CUADRO 3}

Características de los elementos florales de las dos poblaciones de canelos estudiadas. Characteristics of the floral elements of the two canelos populations studied.

\begin{tabular}{|l|rr|rr|}
\hline $\begin{array}{l}\text { Elementos florales } \\
\text { (Valores promedios) }\end{array}$ & \multicolumn{2}{|c|}{ Canelo común (C.V.) } & \multicolumn{2}{|c|}{ Canelo variante (C.V.) } \\
\hline Número de flores por inflorescencia $\left(^{*}\right)$ & 19.90 & $(20.71)$ & 15.80 & $(13.42)$ \\
Número pedúnculos por inflorescencia (n.s.) & 4.20 & $(21.90)$ & 4.70 & $(20.18)$ \\
Número de pétalos por flor (**) & 8.33 & $(8.25)$ & 14.45 & $(5.82)$ \\
Número de sépalos por flor (n.s.) & 2.04 & $(4.02)$ & 2.07 & $(5.59)$ \\
Número de carpelos por flor (*) & 9.01 & $(13.06)$ & 6.91 & $(8.34)$ \\
Número de estambres por flor $(* *)$ & 21.29 & $(25.36)$ & 34.91 & $(5.44)$ \\
\hline
\end{tabular}

$(* *) \quad=$ significativo $(p<0.05)$.

$(*) \quad=$ M uy significativo $(p<0.001)$.

(n.s.) $=$ no significativo.

(C.V.) = coeficiente de variación.

rrollo del área foliar, le disminuye la superficie de evapotranspiración (Stocker 1956, Silva y A cevedo 1984, Larcher 1995) y se asocia con la escasa disponibilidad de agua edáfica de su hábitat, lo que no sucede con el canelo común que crece junto a M irtáceas en suelos muy húmedos y próximos a cursos de agua. Por otro lado, el mayor carácter esclerófilo de las hojas del canelo variante se debería a la gran densidad de células mesofílicas, característica que reduciría el efecto, a nivel celular, de las elevadas tasas de transpiración y a un mayor desarrollo de la vaina del haz conductor, lo que mejoraría la capacidad de resistencia mecánica y evita el colapso de los tubos conductores. A Iberdi et al. (1974) y Alberdi (1995) señalan que el mayor índice de esclerofilia obtenido en hojas de 53 especies nativas siempreverdes está asociado a problemas de balance hídrico. En los prolongados períodos secos de la región, podría producirse una alta evapotranspiración que no se compensaría por la reducida disponibilidad de agua edáfica. La situación es inversa en el hábitat del canelo común, donde el suelo siempre dispone de suficiente agua.

El mayor número de estomas del canelo variante (cuadro 2) es característico de especies que provienen de lugares más secos (Ketellaper 1963, K ramer 1974, Larcher 1980, Donoso 1981a, Strasburger 1987), ratificando la condición más seca de este hábitat.

c) Flores. El número de pétalos y número de estambres por flor presentan diferencias muy sig- nificativas, mientras que el número de flores por inflorescencia y el número de carpelos por flor presentan diferencias sólo significativas $(p<0.05)$ y el número de pedúnculos por inflorescencia y número de sépalos por flor no son diferentes (cuadro 3).

Las diferencias en los elementos florales se usan como indicadores de diferenciación genecológica, porque tienen un corto período de vida en el árbol, por lo tanto están poco expuestas a las modificaciones medioambientales y son menos susceptibles a una variación de tipo plástica (Stebbins 1950)

El gráfico poligonal, constituido con las características florales, muestra una clara diferencia entre el canelo común y el canelo variante (fig. 3). Debido a que están involucrados elementos reproductores se puede atribuir a una variación de tipo genecológica, producto probablemente de la selección natural, que actúa sobre la variabilidad natural de las poblaciones, seleccionando a los genotipos mejor adaptados al medio local (Stebbins 1950, Heslop-Harrison 1964, Millanao 1984).

En el cuadro 4 se muestra que hay una gran variación entre procedencias de canelo, en el número de semillas por kilo. Sin embargo, es notable la diferencia que presenta el canelo variante en relación no sólo con el canelo común de Bullileo, sino con todas las procedencias. Se trataría de las semillas más grandes encontradas en la especie. El tamaño mayor de la semilla puede interpretarse como una adaptación a la sequedad del suelo que 


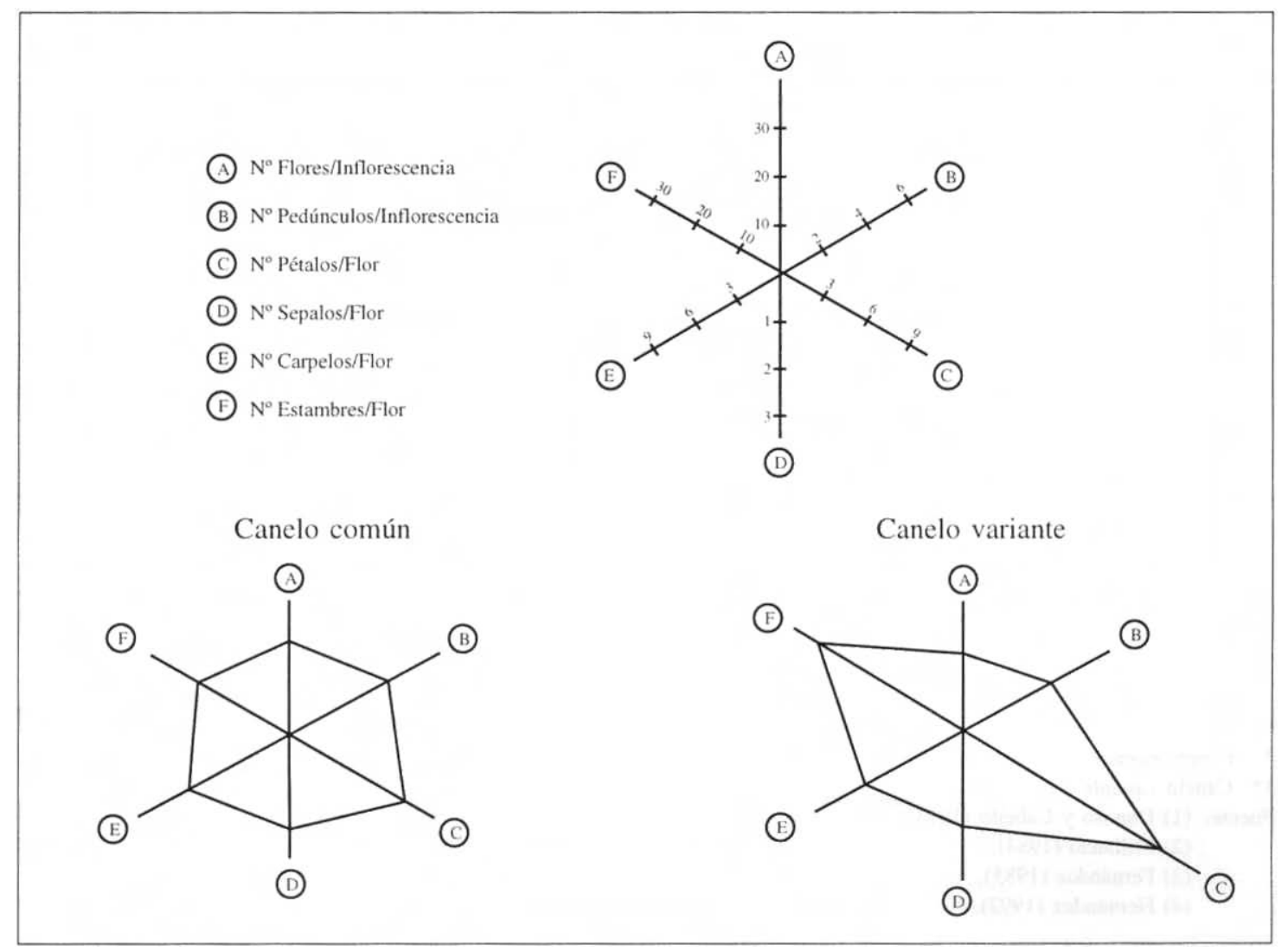

Figura 3. Gráficos poligonales de las características florales para ambos canelos.

Polisonal diagrams of the floral characteristics of both canelos.

se produce en esta región de clima mediterráneo, bajo las condiciones de hábitat de buen drenaje en que vive esta variante de canelo. EI mayor tamaño de las semillas le permite desarrollar, después de germinar, un sistema radicular más fuerte, que es capaz de profundizar rápidamente en busca del agua que se encuentra a mayor profundidad; a medida que avanza el período seco esto es válido para el período de establecimiento, no para toda la vida del árbol (Salisbury, 1942, Stebbins 1950, Baker 1972). La misma adaptación se encuentra en Nothofagus obliqua en su distribución septentrional (Donoso 1979) y parece ser común en varias especies propias de climas más húmedos que se desarrollan en climas mediterráneos en Nothofagus de distribuciones más ecuatoriales, como Nothofagus alpina y Nothofagus dombeyi así como también en Laurelia sempervirens (D onoso 1987).

Características ecofisiológicas. La densidad estomática, la tasa de transpiración momentánea y por ende la tasa de transpiración relativa (cuadro
5) fue superior $(p<0.5)$ en el canelo variante que en el canelo común, ratificándose el carácter xerofítico de los primeros. En éstos, los numerosos estomas pueden permanecer cerrados mientras el suelo carezca de agua. Por el contrario, el adecuado abastecimiento hídrico de la planta provoca la apertura estomática y una tasa de transpiración más elevada que el de las especies no xerofíticas (Stocker 1956. Weinberger et al. 1972, Larcher 1995). En este trabajo las mediciones de transpiración fueron realizadas en laboratorio (temperatura ambiental $22^{\circ} \mathrm{C}$ y humedad relativa del $57 \%$ ) en hojas de ramas saturadas. Los parámetros considerados hasta aquí y relacionados con la resistencia constitucional contribuyen a evitar la deshidratación. Conjuntamente con las diversas adaptaciones estructurales que contribuyen a reducir la pérdida de agua, el citoplasma celular del canelo variante puede soportar deshidratación hasta un $28.66 \%$ del agua celular (figura 4) sin sufrir daños definitivos (cuadro 5). La resistencia plasmática del canelo variante fue superior a la del canelo 


\section{CUADRO 4}

Número de semillas por kilogramo, de diferentes procedencias de Drimys winteri, incluyendo las del estudio. Number of Drimys winteri seeds per kilogram, from different origins including the study.

\begin{tabular}{|l|c|c|}
\hline Procedencia & $\begin{array}{c}\text { Número semillas/kilos } \\
\text { (Valores promedios) }\end{array}$ & Altura (m s.n.m.) \\
\hline La Dormida (Santiago) (3) & 233.135 & 600 \\
Bullileo (VII Región (1) * & 240.000 & 700 \\
Bullileo (VII Región (4) ** & 110.248 & 700 \\
Villarrica (IX Región) (3) & 191.000 & 400 \\
Piren Alto (IX Región (2) & 167.000 & 750 \\
Colligual (X Región) (3) & 187.248 & 600 \\
Huape (X Región) (2) & 335.788 & 20 \\
Fundos (X Región) (3) & 361.477 & 0 \\
Jardín Botánico' (X Región) (3) & 319.880 & 0 \\
Jardín Botánico² (X Región) (4) & 207.973 & 0 \\
Llancacura & 319.880 & 50 \\
\hline
\end{tabular}

* Canelo común.

** Canelo variante.

Fuente: (1) Donoso y Cabello (1978).

(2) Millanao (1984).

(3) Fernández (1985).

(4) Hernández (1992).

\section{CUADRO 5}

Variables ecofisiológicas de las dos poblaciones de canelos estudiados.

Ecophysiological variables of the two canelo populations studied.

\begin{tabular}{|l|c|c|}
\hline Características ecofisiológicas & Canelo común & Canelo variante \\
(Valores promedios) & & \\
\hline Déficit crítico subletal (\%) (A.R.) & 20.96 & 28.66 \\
Transpiración momentánea (mg $\left.\mathrm{H}_{2} \mathrm{O} / \mathrm{g} / \mathrm{min}\right)(* *)$ & 1.22 & 1.86 \\
Transpiración relativa (\%) (*) & 15.22 & 22.90 \\
\hline
\end{tabular}

A.R. = A nálisis de regresión.

$(* *)=$ Muy significativo $(p<0.001)$.

común en un $27.1 \%$. Millanao (1984) también estudió la resistencia plasmática del canelo de dos poblaciones $\left(39^{\circ} 20^{\prime} \mathrm{S}\right)$ que crecen en diferentes hábitats y encontró que en los individuos que crecen en suelos bien drenados de la Cordillera de la Costa a 750 m s.n.m. tienen un citoplasma más resistente a la pérdida de agua (35.69\%) que los que crecen en suelos tipo hualves junto a $M$ irtáceas a 20 m s.n.m. (26.34\%). Es decir, la mayor capacidad de resistencia plasmática de los individuos que crecen en suelos de gran permeabilidad $(26.2 \%)$ respecto a suelos muy hidratados es semejante a la encontrada en las dos poblaciones de la precordillera andina de Parral. Por el contrario, W einberger et al. (1972) entregan el valor para el genotipo $(55 \%)$ y establecen que, en general, las especies de la zona esclerófila con clima de tipo mediterráneo presentan menor resistencia plasmática que las especies de la pluvioselva subantártica de clima frío y muy húmedo. El mayor valor de la 


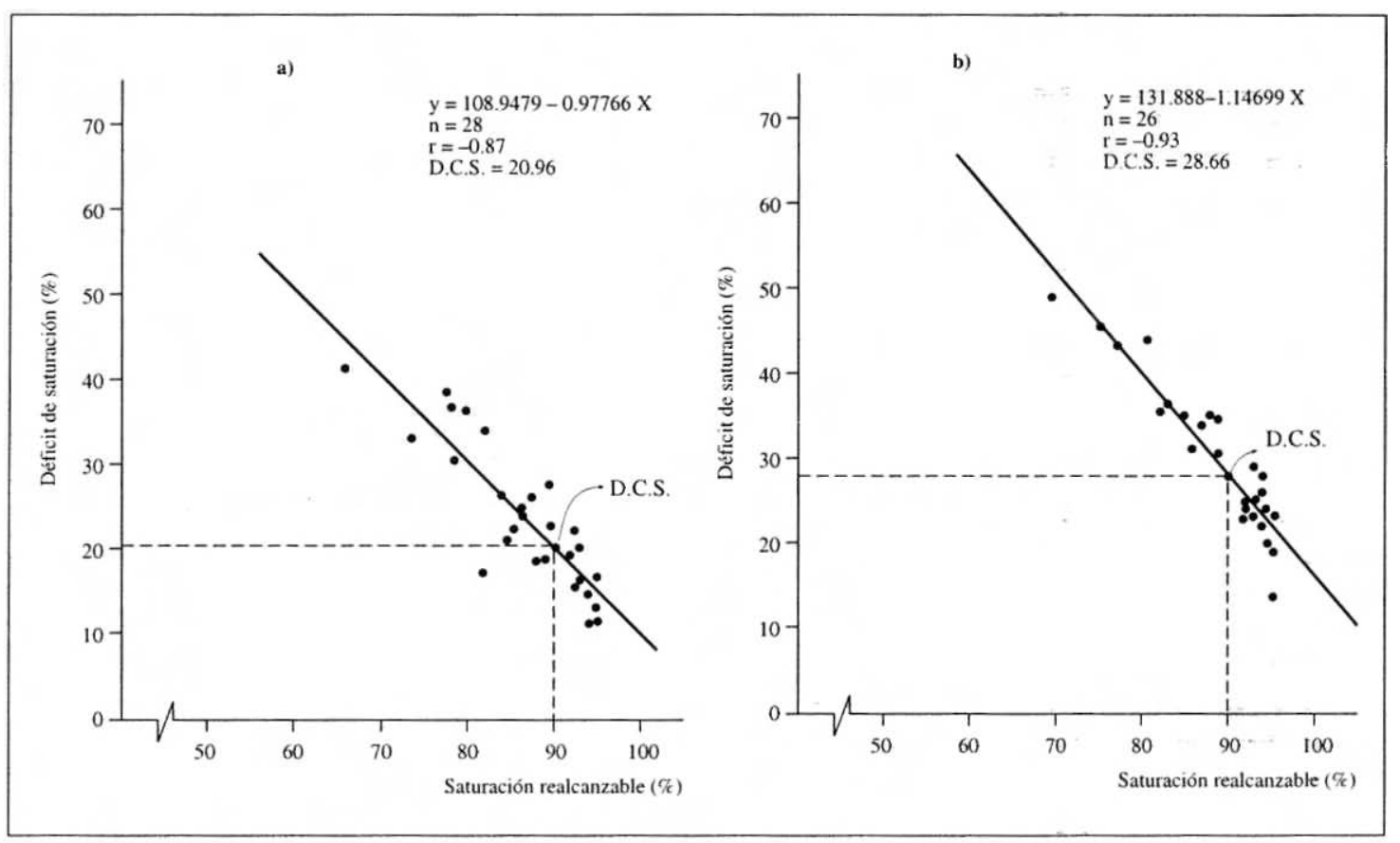

Figura 4. Déficit crítico subletal (D.C.S.): a) canelo común, b) canelo variante.

Critical subletal deficit (C.S.D.): a) common canelo, b) variante canelo.

resistencia plasmática de las especies subantárticas se asociaría a la sequía fisiológica provocada por suelos fríos (Stocker 1956, Larcher 1995).

El comportamiento de las características ecofisiológicas analizadas es coherente con el análisis de las características morfológicas de las hojas, incluyendo el tamaño de las semillas, en el sentido de que representa la adaptación a hábitat más seco del canelo variante en comparación con el canelo común.

Características químicas. Visualmente las hojas de ambas procedencias presentan claras diferencias, reflejadas en la ausencia del color glauco en el envés de las hojas del canelo variante, característica típica de esta especie y muy particular y notoria en hojas del canelo común.

La cuantificación del contenido de ceras epicuticulares arrojó como resultado una cantidad significativamente mayor $(p<0.05)$ en el canelo común $\left(0.38 \mathrm{~g} / \mathrm{dm}^{2}\right)$ que en el canelo variante $(0.23$ $\left.\mathrm{g} / \mathrm{dm}^{2}\right)$, principalmente en el envés de la hoja. La mayor cantidad de ceras epicuticulares del canelo común bloquearían la transpiración cuticular sólo si la humedad relativa ambiental no fuera lo suficientemente alta como para embeberla. En caso contrario no afectarían la pérdida de agua. Según Strasburger (1986), las ceras en el envés actuarían sobre la cutícula, disminuyendo las pérdidas de agua por transpiración cuando no están embebidas. Esta mayor cantidad de ceras sobre la cutícula en el envés del canelo común se relacionaría con la menor tasa de transpiración evidenciada en éste. Por otro lado, el canelo variante debería tener más ceras epicuticulares que el común, por el hecho de que al crecer en un hábitat más seco requerirá disminuir su tasa de transpiración para compensar la menor disponibilidad de agua en el suelo, comparada con la del canelo común que crece en hualves.

Por ahora no disponemos de una explicación fisiológica, sin embargo subsiste la clara diferenciación morfológica y química entre ambos tipos de canelo.

Los cromatogramas de flavonoides de las hojas de canelo común y del variante son diferentes (fig. 5). Las manchas número uno a la seis pueden considerarse iguales. La diferencia la marcan las manchas número siete y ocho, que sólo están presentes en el cromatograma del canelo variante, lo que es confirmado por la mayor transmitancia que muestra la curva del canelo variante y la diferencia en los puntos de inflexión de ambas curvas (fig. 6).

$\mathrm{Si}$ se considera que los flavonoides son. compuestos característicos, esta diferencia muestra 
a)

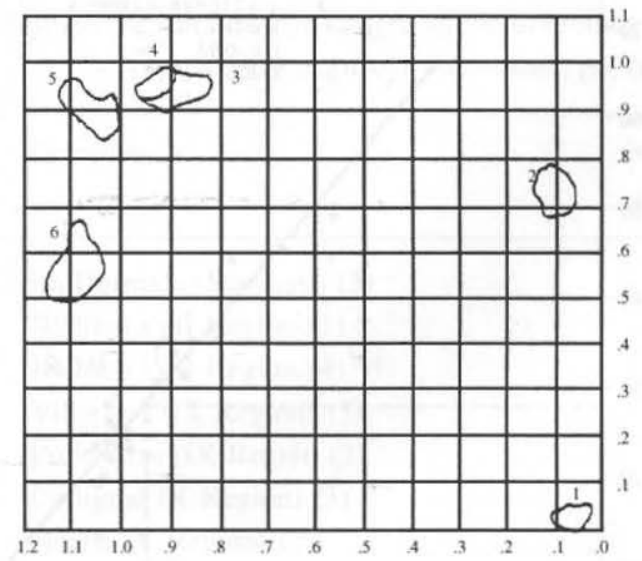

b)

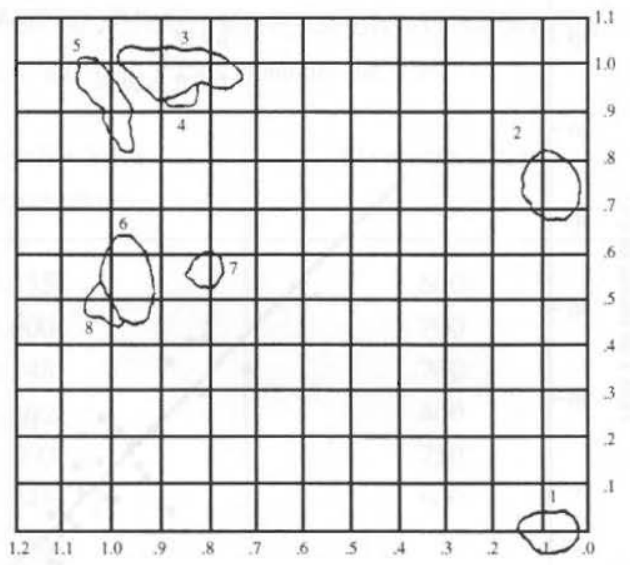

Figura 5. Cromatografía de flavonoides: a) canelo común, b) canelo variante.

Flavonoid chromatography: a) common canelo, b) variant canelo.

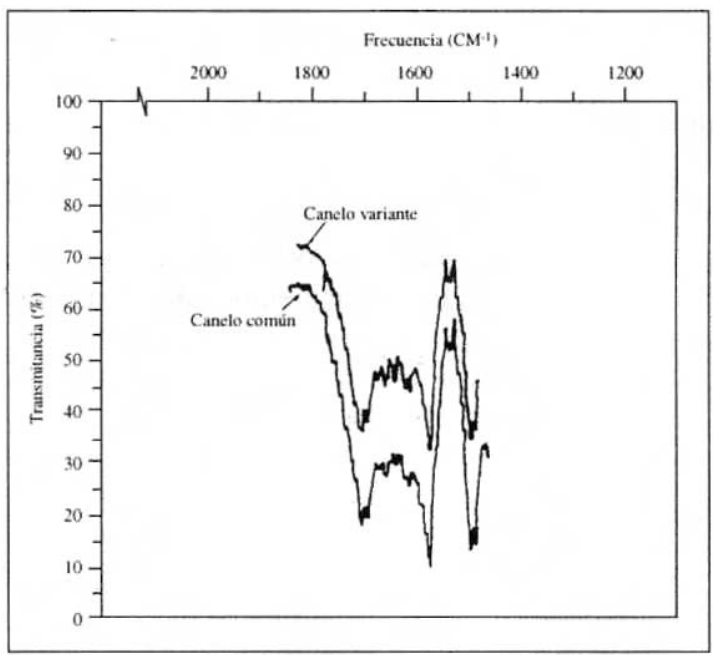

Figura 6. Curvas de transmitancia del canelo común y del canelo variante.

Transmittance curves of the common and of the variant canelo.

modificaciones en el complejo de flavonoides presentes. Al respecto se ha sugerido que flavonoides del tipo flavonas y flavonoles presentes en hojas absorben intensamente longitudes de ondas en la región UV. Algunos flavonoides como quercitina y apigenina han sido implicados en la regulación endógena del transporte polar de auxinas y juegan un rol ecofisiológico de gran importancia (Taiz y Zeiger 1991).

\section{CONCLUSIONES}

La presencia de agua en la superficie del suelo y la dominancia de las Mirtáceas en el hábitat de canelo común contrasta con el hábitat del canelo variante, que es claramente más seco.

Estas características del hábitat están claramente correlacionadas con las características morfológicas de las hojas, el tamaño de las semillas y el comportamiento ecofisiológico de los dos tipos de canelo, confirmando todas ellas la mayor adaptación del canelo variante a una condición de hábitat con mayores restricciones de humedad.

Estas diferencias entre poblaciones contiguas de una misma especie sugieren la ocurrencia de una diferenciación genecológica que se ve corroborada por la diferenciación significativa entre los elementos florales, entre los cromatogramas de fenoles y en relación con la presencia o ausencia de una substancia irritante o picante en las hojas de ambas poblaciones. Por lo tanto, se sugiere en este trabajo que existen suficientes evidencias de la ocurrencia de dos ecotipos contiguos de Drimys winteri en la localidad precordillerana de Bullileo.

\section{BIBLIOGRAFIA}

A BBOT, D., R. ANDREWS. 1970. Introducción a la cromatografia. Editorial Alhambra S.A. Madrid, $121 \mathrm{pp}$.

ALBERDI, M., P. WEINBERGER, M. OLIVA, M. ROMERO. 1974. Ein Beitragzur chemischen Kennzeichnung des 
Skleromorphie-Grades von Blättern immiergrüner Gehölze, en Beiträge zur und Biologie der Pflanzen 50(2): 305-320.

A LBERDI, M. 1995. Ecología de especies leñosas de los bosques higrófilos templados de Chile: resistencia a la sequía y bajas temperaturas. En ARMESTO, J., C. VILLAGRAN y M. K. ARROYO, (eds.). Ecología de los bosques nativos de Chile. Monografías. Editorial Universitaria, pp. 275-300.

BAKER, H.G. 1972. "Seed weight in relation to environmental conditions in California", Ecology $53 \mathrm{~N}^{\circ} 6$.

BELL, C. 1968. Variación y clasificación de las plantas. Serie fundamento de la Botánica. Herrero Hermanos Sucesores, S.A. M éxico, $142 \mathrm{pp}$.

CORVALAN, P. 1986. Informe de avance: El canelo: una alternativa de desarrollo para la $X$ Región. Stgo., Ministerio de Agricultura - Universidad de Chile.

DI CASTRI, F., E. HAJEK. 1976. Bioclimatología de Chile. Vicerrectoría Académica de la Universidad Católica de Chile, $128 \mathrm{pp}$.

DOMINGUEZ, X. 1975. Cromatografía en papel y en capa delgada. Unión Panamericana. Dpto. de Asuntos Científicos. Serie Química. Monografía, pp. 16-80.

DONOSO, C. 1974. Dendrología, árboles y arbustos chilenos. Facultad de Ciencias Forestales, Universidad de Chile, $\mathrm{M}$ anual $\mathrm{N}^{\circ} 2.29$ p.

DONOSO, C. 1979. "Variación y tipos de diferenciación en poblaciones de Roble (Nothofagus obliqua (M irb. Oerst.)", Bosque 3(1): 1-14.

DONOSO, C. 1981a. Ecología forestal. El bosque y su medio ambiente. Facultad de Ciencias Forestales. Universidad A ustral de Chile, 369 pp.

DONOSO, C. 1981b. Tipos forestales de los bosques nativos de Chile. Doc. de trabajo $\mathrm{N}^{\circ} 38 \mathrm{CONAF}, \mathrm{FAO}, \mathrm{DP} / \mathrm{CHI} /$ $76 / 003$.

DONOSO, C. 1987. "Variación natural en especies de Nothofagus en Chile", Bosque 8(2): 85-97.

DONOSO, C. 1993. Bosques templados de Chile y Argentina. Variación, estructura y dinámica. Ecología forestal. Edit. Universitaria, $484 \mathrm{pp}$.

DON OSO, C., L. LANDRUM. 1976. Nothofagus leoni: Hibridación e introgresión en poblaciones de Nothofagus obliqua $y$ Nothofagus glauca. Bol. Tecn. $\mathrm{N}^{\circ} 36, \mathrm{Fac}$. Ciencias Forestales, Universidad de Chile.

FERNANDEZ, J. 1985. Propagación germinativa y vegetativa de Drimys winteri J.R. y G. Forster. Tesis Ingeniero Forestal, Fac. de Cs. Forestales, Universidad Austral de Chile, Valdivia, $80 \mathrm{pp}$.

HARBERD, D.J. 1956. "The within population variance in genecological trials", New Phytol 56: 269-280.

HERNANDEZ, M. 1992. A nálisis de la variación de dos poblaciones contiguas de Drimys winteri (Forst.) en la precordillera andina de la VII Región. Tesis, Ingeniero Forestal, Fac. de Cs. Forestales, Universidad Austral de Chile, Valdivia, $78 \mathrm{pp}$

HESLOP-HARRISON, J. 1964. "Forty years of Genecology", Adv. Ecol. Res. 2

HEY W OOD, V. 1967. Taxonomía vegetal. Ed. A Ihambra S.A., M adrid, $102 \mathrm{pp}$.

HOFFMANN, A. 1982. Flora silvestre de Chile. Zona Austral. Ed. Fundación Claudio Gay, Santiago.

KeTELlAPER, H. 1963. "Stomatal Physiology", Rev. Pant Physiology 144: 249-270.

KRAMER, P. 1974. Relaciones hídricas de suelo y plantas una sintesis moderna. Centro Regional de Ayuda Técnica. M éxico, $538 \mathrm{pp}$.
LARCHER, W. 1980. Oekologie der Pflanzen. 3 Auflage 181. A bb. 54 Tabellen. Verlag UImer Stuttgart, $272 \mathrm{pp}$.

MILLANAO, D. 1984. Diferenciación genecológica de dos poblaciones de Drimys winteri Forst. (IX y X Región, Chile). Tesis Ingeniero Forestal, Fac. de Cs. Forestales, Universidad A ustral de Chile, Valdivia, $74 \mathrm{pp}$.

MUÑOZ, C. 1959. Sinopsis de flora chilena clave para la identificación de familia y género. Editorial Universitaria, $830 \mathrm{pp}$.

M UÑ OZ, M. 1980. Flora del Parque Nacional Puyehue. Editorial Universitaria S.A Santiago.

NIE, N., C. HULL, J. JENKIS, K. STEIBRENNER, D. BENT 1975. Statistical Package for the Social Sciences. M CGrawHill Book Company, $675 \mathrm{pp}$.

OSTLE, B. 1968. Estadística aplicada; técnica de la estadística moderna, cuándo y dónde aplicarla. Centro Regional de A yuda Técnica Limusa-Wiley, M éxico, 629 pp.

RODRIGUEZ, R., O. MATTHEI, M. QUEZADA. 1983. Flora arbórea de Chile. Concepción, Chile, Universidad de Concepción, 408 pp.

RODRIGUEZ, R., M. QUEZADA. 1991. "Nueva combinación en Drimys J. R. y G. Forster (Winteracea) de Chile". Gayana B ot. 48 (1-4): 111-114.

SALISBURY, E. J. 1942. The Reproductive Capacity of Plants. London, Bell.

SILVA, H., E. ACEVEDO. 1984. A daptaciones anatomorfológicas foliares al déficit hídrico en Atriplex repannda Phil", Rev. Chilena de Historia Natural 57: 69-78.

SOKAL, R., F. ROHLF. 1979. Biometría; principios y métodos estadísticos en la investigación biológica. H. Blume Ediciones, Madrid, $832 \mathrm{pp}$.

SPURR, Z.H., B.V. BARNES. 1973. Forest ecology. The Ronald Press Co., New Y ork.

SPURR, S., B. BARNES. 1980. Forest ecology. Third edition, John Wiley \& Sons, $687 \mathrm{pp}$

STEBBINS, G. 1950. Variation and evolution in plants. Columbia University Press, New Y ork.

STEUBING， L. 1965. Pflanzenokologisches Praktikum; Methodern und Geräte Zur. Bestimmung Wichtiger Standorfsfacktoren. Paul Parey, Berlin, 262 pp.

STOCKER, O. 1956. Die Dürreresistenz. En: Handbuch der Pflanzenphysiologie. Bol. 3: 696-741.

STRA SBURGER，E. 1986. Tratado de Botánica. $7^{\star}$ ed. Española, Barcelona, M anuel M arín, 1098 pp.

TAIZ, L., E. ZEIGER. 1991. Plant physiology. The Benjamin Cumming Publishing Company, Inc. $559 \mathrm{pp}$.

TA JAN, P.A. 1989. Crecimiento y resistencia a la sequía de ocho procedencias de Raulí (Nothofagus alpina (Poepp. et Endl.) Oerst.), durante el segundo período vegetativo de V aldivia. Tesis, Ingeniero Forestal, Fac. de Cs. Forestales, Universidad Austral de Chile, Valdivia.

TORRES, H. 1971. Maderas. Corporación Chilena de la Madera. Santiago, Chile. Prensa Latinoamericana, $270 \mathrm{pp}$

TRONCOSO, A., C. VILLAGRAN, M. MUÑOZ. 1980. "Una nueva hipótesis acerca del origen y edad del bosque Fray Jorge (Coquimbo, Chile)", Bol. Mus. Nacional Hist. Nat. 37: 117-152.

URBAN, O. 1934. Botánica de las plantas endémicas de Chile. Soc. Imp. y Litografía "Concepción". Concepción.

WEINBERGER，P., M. ROMERO，M. OLIVA. 1972. "Ein methodischer Beitrag Zur Bestimmung", Flora 161: 555561. 